ORIGINAL ARTICLE

\title{
Comparative Efficacy of Progressive Resistance Exercise and Biomechanical Ankle Platform System on Functional Indices of Children with Cerebral Palsy
}

\author{
Faderera Adepoju ${ }^{1}$, Talhatu Hamzat ${ }^{2 *}$, Olusegun Akinyinka ${ }^{2}$
}

\section{OPEN ACCESS}

Citation: Faderera Adepoju, Talhatu Hamzat, Olusegun Akinyinka. Comparative Efficacy of Progressive Resistance Exercise and Biomechanical Ankle Platform System on Functional Indices of Children with Cerebral Palsy. Ethiop J Health Sci 2017;27(1):11-16. doi:

http://dx.doi.org/10.4314/ejhs.v27i1.3

Received: August 27, 2016

Accepted: August 29, 2016

Published: December 1, 2017

Copyright: Faderera A, et al. This is an

open access article distributed under the terms of the Creative Commons

Attribution License, which permits unrestricted use, distribution, and reproduction in any medium, provided the original author and source are credited.

Funding: Nil

Competing Interests: The authors

declare that this manuscript was approved

by all authors in its form and that no

competing interest exists.

Affiliation and Correspondence:

${ }^{1}$ Department of Physiotherapy,

University College Hospital, Ibadan,

Nigeria

${ }^{2}$ Department of Physiotherapy, College of Medicine, University of Ibadan, Nigeria

${ }^{3}$ Department of Paediatrics, College of Medicine, University of Ibadan, Nigeria Email: tkhamzat@com.ui.edu.ng; talkzat@yahoo.com

\section{ABSTRACT}

BACKGROUND: Progressive Resistance Exercise (PRE) and Biomechanical Ankle Platform System (BAPS) are two of the protocols available in managing children with Cerebral Palsy $(C P)$. The comparative effects of these modalities on selected functional indices of ambulatory type $C P$ were the focus of this study METHODS: Twenty-eight children with hemiplegic or diplegic $\mathrm{CP}$ receiving care at a tertiary health facility in Ibadan were consecutively recruited. They were systematically assigned into two intervention groups. Namely PRE, BAPS. Both groups received intervention twice weekly for 16 weeks. At baseline, 8 and 16 weeks of intervention balance and functional mobility were assessed using Berg Balance Scale (BBS) and modified timed-up-and-go test (TUG) respectively. Chi-square, Fisher's Exact tests, One way and repeated measures ANOVA were carried out. Level of significance (p) was set at 0.05 .

RESULTS: There were significant differences in the functional indices of participants in the BAPS group at the end of the intervention ( $p<0.05)$. The two groups (BAPS and PRE) were not significantly different at baseline and 8 and 16 weeks $(p>0.05)$. All outcome measures increased in both groups from baseline to the end of the intervention period.

CONCLUSION: The two intervention protocols demonstrated improvements in the areas assessed. Comparatively, both PRE and $B A P S$ could be used to promote function in CP.

KEYWORDS: Cerebral Palsy, Biomechanical Ankle Platform System, Progressive Resistance Exercise, Functional Indicesno

\section{INTRODUCTION}

Cerebral palsy (CP) is the most common developmental disorder associated with lifelong motor impairments and disabilities (1). Voluntary motor control impairments in children with $\mathrm{CP}$ result in disorders of balance control (2). Balance dysfunctions often pose a great challenge in the ambulatory types of cerebral palsy, namely hemiplegia and diplegia. These challenges become more noticeable 
when the affected child is performing activities of daily living that require antigravity postural control; particularly standing and walking $(3,4)$. Poor balance control in cerebral palsy could be due to factors such as muscle weakness, reduced contraction of agonist muscles and loss of selectivity in neuromuscular output which could cause co-contraction of agonists and antagonists (5,6).

Managing cerebral palsy encompasses training motor tasks, facilitating functional movements and optimising the child's potential while performing tasks in a variety of environmental settings and maintaining antigravity postural control (7). The burden of caring for children with $\mathrm{CP}$ is considerable with the care-givers often actively involved. Rehabilitation of CP must therefore be caregiver-centred because evidence has shown that quality of life of care- givers is negatively affected as they have to combine their activities of daily living with effective management of their children $(8,9)$. Using affordable and easily accessible equipment could promote familycentred care thereby reducing care-giver burden in terms of time, costs and frequency of clinic visits.

Biomechanical Ankle Platform System (BAPS) is closed-chain exercise equipment which uses the body weight as resistance (12). With the feet positioned on the board, the patient shifts weight laterally and antero-posteriorly while attempting to control the ankle and maintain balance (12). Strength training in various forms such as task-specific exercises and progressive resistance exercise have been shown to increase muscle strength and improve function in children with CP $(10,11)$. Progressive resistance exercise in lower limbs resulted in improved balance, and optimal results were reported when training was individualised in functional postures ain which intensity was progressively increased $(10,11)$. Evidence has shown that BAPS is effective in rehabilitation of lower limb injuries $(11,12,13)$. However, its efficacy in enhancing functional performance in children with $\mathrm{CP}$ appears not to have received attention; there is dearth of published literature on the subject. This study thus addressed the following research questions:

a) Would BAPS significantly improve some functional abilities in children with CP? b) Would there be a significant difference in the comparative efficacy of PRE and BAPS on functional indices (balance, functional mobility) of children with CP?

\section{METHODS}

Participants: Twenty-eight children aged between 4 and 12 years with hemiplegic or diplegic $\mathrm{CP}$ were consecutively recruited from outpatient paediatric neurology clinic of a tertiary health facility and special children centres in Ibadan. Diagnosis of cerebral palsy was made by a paediatric neurologist. The participants were further assessed by a licensed physiotherapist to ensure that they met the inclusion criteria. Eligibility criteria included: 1) spasticity in hip and knee flexors, extensors less than or equal to 2 on MAS (16); 2) ability to comprehend instructions and communicate verbally and 3) absence of co-morbid conditions such as epilepsy and mental retardation.

Procedure: Ethical approval of the University of Ibadan/University College Hospital Institutional Review Committee was obtained before the commencement of this study (UI/EC/09/0083). Informed consent of the children was obtained by proxy after explanation of the procedure to the their care-givers. The children's assents were also obtained. Consenting participants were assigned into one of the intervention groups $(1,2)$ using random assignment through a lucky dip (participant picked one piece of paper from a bowl containing two pieces of paper). The first two consenting participants picked 1 and 2 while subsequent participants were assigned alternately to the two groups.

A licensed physiotherapist who was blinded to the intervention the recipient received carried out the pre- and post-intervention assessments of the parameters. Treatment interventions were carried out by one of the authors (FAA).

The Gross Motor Function Classification System (GMFCS) was used to classify motor function in the participants. It consists of five ordinal levels. Each level describes the child's present abilities and limitations in motor function (14).

DOI: http://dx.doi.org/10.4314/ejhs.v27i1.3 
Berg Balance Scale (BBS) was used to assess balance in the participants. It consists of 14 items, and performance is scored on a 5-point scale from 0-4 with the maximum score of 56 points (15).

The Modified Ashworth scale (MAS) was used to assess muscle spasticity. This is a 6-point ordinal scale that describes muscle tone and movement at the affected limbs (16).

The modified 'timed up to go' test (TUG) for children was used as a measure of functional mobility. The procedure was carried out as outlined by Williams et al (17):

A chair was positioned three meters away from a wall such that it was stable and did not shift when the participant moved from sitting to standing. The participant sat on the chair with his feet flat on the floor and knees at 90 degrees. On instruction, the participant stood up and walked the 3 meters at his/her regular pace, touched a drawing on the wall; and then walked back to the chair and sat down. Timing started when the participant stood up and stopped when the participant sat down again on the chair after covering six meters (to and from the chair). This ensured that only the movement time was measured. Three trials were carried out and the average score of the trials was used for data analysis. Walking speed was computed by dividing the distance covered (6 metres) by the time taken.

Treatment/Intervention groups: The exercises were prescribed using the recommended protocols $(18,19)$ which identified closed chain exercises to target major muscle groups of lower limbs. Closed-chain exercises can involve concentric, eccentric or isometric action which load bones, joints and non-contractile soft tissues. cocontraction of muscles is severely impaired in $\mathrm{CP}$; closed chain exercises also stimulate mechanoreceptors around and in the joints, thereby stimulating muscle contraction. Performance of these exercises in functional position of standing aids mobility skills.

Group 1-Strengthening exercise group: The major muscle groups of the lower limbs have been reported to contribute immensely to closed chain exercises involving the lower limbs $(11,12)$. Each exercise bout comprised ten repetitions. The exercises are described below:
A. Wall slides: This exercise involves the hip flexors and extensors plus knee flexors and extensors. In standing, the participant rested his back against the wall, with both feet firmly placed on the floor and about shoulder width apart. He/she then slid the trunk down the wall by flexing the hips and knees and consecutively up the wall by extending the hips and knees. Five bouts repetitions of the exercise were carried out, and progression was done by increasing the bouts and also introducing of arm motions of flexion and abduction of the shoulders. Further progression was done by each participant holding weights of $0.5 \mathrm{~kg}, 1 \mathrm{~kg}$ and $2 \mathrm{~kg}$ as required.

B. Bridging: Hip extensors, knee flexors and dorsiflexors were involved in bridging. With the participant in supine lying position, the knees and hips were bent such that the soles of the feet were flat on the mat while the pelvis was elevated. The position was sustained for ten seconds and a total of three bouts were carried out at each exercise session. Progression was introduced when the participant could perform bridging with one leg.

C. Side stepping: All muscles of the lower limbs were involved in this exercise. The participant stood with his side facing the staircase, and then stepped up and down the first step (height $17 \mathrm{~cm}$; width $29.5 \mathrm{~cm}$ ). Three bouts were carried at each exercise session. Progression was achieved by increasing the number of steps when the participant was able to perform the required bouts for each session.

Group 2: Biomechanical ankle platform system group: The BAPS was placed on the floor, each participant stood with both feet on the balance board and then shifted his/her body weight forward and backward and from side to side for the perturbation exercises. Participants were initially allowed to hold on to wall bars as support; progression of exercise was determined by the participants carrying out the perturbation exercises without holding on to any support. Perturbations of the balance board were carried out by each participant at his/her individual pace, but they 
were encouraged to perform 50 repetitions per session (11).

Participants came in at various levels of motor function; individualized Progressive Resistance Exercise (PRE) principle of repetition maximum was adopted. As participants capabilities improved, repetitions were increased to continually overload muscles with this leading to adaptive changes. Furthermore, starting doses for the exercises were individualized and whatever difference(s) that would have been due to the fact that two types of $\mathrm{CP}$ were involved in the study was adjusted for using appropriate statistical analysis.

Data analyses: Data were analysed using the SPSS package 15.0 for windows (SPSS Inc. Chicago, USA). Descriptive statistics of mean and standard deviation were used to present the age, TUG scores, Chi-square test was used to present topography and Fisher's Exact test was used to present the MAS of the participants. One-way ANOVA was used to compare baseline and 8 weeks plus baseline and 16 weeks scores in the two groups. Repeated measures ANOVA was used to compare walking speed and BBS scores in the BAPS group from baseline to 16 weeks. Level of significance (p) was set at 0.05 .

\section{RESULTS}

Physical and clinical attributes of the participants are presented in Table 1 . There was no significant difference $(p>0.05)$ in the PRE and BAPS groups at baseline. BBS scores and walking speed of participants in the BAPS group improved significantly $(p<0.05)$ from baseline to the end of the intervention, thereby corroborating the efficacy of BAPS in the management of children with CP (Table 2).

Table 1: Physical and Clinical Characteristics of participants $(\mathrm{N}=28)$

\begin{tabular}{llll}
\hline Variable & BAPS & PRE & p-value \\
\hline AGE (Yrs) & $9.1 \pm 2.9$ & $7.6 \pm 2.1$ & 0.128 \\
MAS & & & \\
0 & $5(33.3)$ & $4(30.8)$ & \\
1 & $6(40.0)$ & $3(23.1)$ & \\
$1+$ & $2(13.3)$ & $0(0.0)$ & 0.062 \\
2 & $\mathrm{a} 2(13.3)$ & $6(46.2)$ & \\
TOPOGRAPHY & & & \\
Diplegic & $7(46.7)$ & $6(46.2)$ & \\
Hemiplegic & $8(53.3)$ & $7(53.8)$ & 0.978 \\
TUG & $0.50(0.07)$ & $0.46(0.07)$ & 0.119 \\
\hline
\end{tabular}

Table 2: Repeated measures of ANOVA showing BBS and walking speed of the BAPS group from baseline to 16 weeks

\begin{tabular}{llc}
\hline Time & BBS & W/S \\
& $\ddot{x} \pm$ S.D & $\ddot{x} \pm$ S.D \\
\hline Baseline & $38.67 \pm 8.83$ & $0.50 \pm 0.07$ \\
2 weeks & $38.67 \pm 8.83$ & $0.49 \pm 0.07$ \\
4 weeks & $40.67 \pm 8.39$ & $0.51 \pm 0.07$ \\
6 weeks & $42.65 \pm 8.32$ & $0.52 \pm 0.07$ \\
8 weeks & $44.77 \pm 8.26$ & $0.52 \pm 0.07$ \\
10 weeks & $45.15 \pm 8.13$ & $0.53 \pm 0.06$ \\
12 weeks & $46.00 \pm 7.56$ & $0.53 \pm 0.07$ \\
14 weeks & $46.91 \pm 7.70$ & $0.54 \pm 0.07$ \\
16 weeks & $48.45 \pm 5.94$ & $0.54 \pm 0.07$ \\
p- value & 0.04 & 0.03 \\
\hline BAPS: Biomechanical Ankle Platform System group \\
BBS: Berg Balance Score, W/ S: Walking Speed
\end{tabular}

Table 3: Two One-way ANOVA showing comparison of BAPS and PRE

\begin{tabular}{lcccc}
\hline PERIOD & BAPS & PRE & $\begin{array}{l}\mathrm{F}- \\
\text { value }\end{array}$ & $\begin{array}{l}\mathrm{p}- \\
\text { value }\end{array}$ \\
\hline $\begin{array}{l}\text { BBS: } \\
\text { Baseline - } 8\end{array}$ & $6.53 \pm 2.79$ & $5.27 \pm 1.68$ & 1.73 & 0.202 \\
$\begin{array}{l}\text { weeks } \\
\text { Baseline - 16 } \\
\text { weeks }\end{array}$ & $10.27 \pm 4.84$ & $8.17 \pm 2.48$ & 0.975 & 0.339 \\
$\begin{array}{l}\text { W/ S: } \\
\text { baseline - } 8\end{array}$ & $0.03 \pm 0.04$ & $0.02 \pm 0.01$ & 0.117 & 0.736 \\
$\begin{array}{l}\text { weeks } \\
\text { Baseline - 16 } \\
\text { weeks }\end{array}$ & $0.05 \pm 0.04$ & $0.06 \pm 0.03$ & 0.257 & 0.619 \\
\hline $\begin{array}{l}\text { BAPS: Biomechanical Ankle Platform System group } \\
\text { BBS: Berg Balance Score, W/ S: walking Speed }\end{array}$ &
\end{tabular}

DOI: http://dx.doi.org/10.4314/ejhs.v27i1.3 
Comparative efficacy of BAPS and PRE was not significantly different between baseline and eight weeks plus baseline and 16 weeks $(\mathrm{p}>0.05)$ as shown in table 3.

\section{DISCUSSION}

Participants in this study were children with either type of ambulant cerebral palsy, namely diplegia or hemiplegia. The focus of this research did not include comparison between children with diplegic and hemiplegic cerebral palsy, both groups constituting the ambulant type cerebral palsy. At baseline, there were significant differences in the functional performance of the participants. Statistical adjustments were made using ANCOVA with the type of $\mathrm{CP}$ as the covariate.

Children with ambulatory types of $\mathrm{CP}$ are known to walk with low speed and abnormal kinetics (5,6). Independent and assisted ambulation is therefore a major goal in the management of children with cerebral palsy because it has been associated with their employability, economic status and social integration as they grow older $(5,20,21)$.

Paucity of data on the use of BAPS and strengthening exercises in rehabilitation of children with ambulatory $\mathrm{CP}$ made comparison of our findings with previous ones difficult. There is no consensus on the beneficial effects of strengthening exercise on functional abilities of children with cerebral palsy. Lee et al (22) suggested that strengthening exercises could be a useful method to improve gait function of patients with spastic CP. Conversely, a systematic review concluded that strengthening interventions improved neither strength nor activity in cerebral palsy and did not appear to increase spasticity, adding that there was sparse evidence on the spasticity (23) . More recent studies however reported that strengthening exercise improved muscle strength to greater degree (24) and that controlled ankle plantarflexion strengthening program may lead to improvements in strength and spatiotemporal gait parameters of children with cerebral palsy (25).

Improvements in functional indices following both interventions could have been due increased strength and task specific changes. Learning adaptations result from repetition of tasks and practice; both are fundamental to enhancement of neuroplasticity which underlies all forms of skill learning. BAPS improved balance performance and walking speed of children with hemiplegic and diplegic cerebral palsy over the intervention period. Woollacott et al (26) reported that the changes observed after reactive balance training to improved proprioceptive sensitivity in leg muscles, enhanced synaptic efficacy within the primary motor cortex pathways and higher levels of adaptations at the cerebellum and association cortex.

Most of the studies that evaluated functional performance in children with $\mathrm{CP}$ had single group design. Comparative inference was therefore difficult. This study showed that comparatively, PRE and BAPS were both efficacious but not significantly different in the management of children with ambulatory types of cerebral palsy. Use of BAPS and PRE could enhance functional abilities in children with CP. Use of these readily available, affordable and easily replicable modalities could reduce burden of caring for children with $\mathrm{CP}$ as both are safe for home use under adult supervision.

\section{REFERENCES}

1. Aisen ML, Kerkovich D, Mast J, Mulroy S, Wrench TL, Kay RM. Cerebral palsy: clinical care and neurological rehabilitation. Lancet Neurol 2011; 10: 844-852.

2. Woollacott M and Shuway-Cook A. Postural dysfunction during standing and walking in children with cerebral palsy: what are the underlying problems and what new therapies might improve balance. Neural Plast 2005; 12: 211-219.

3. Damiano DL. Rehabilitation therapies in cerebral palsy: the good, the not as good and the possible. J Child Neurol 2009; 24 (9): 12001204

4. Van der Heide JW and Hadders - Algra MC. Postural muscle dyscoordination in children with cerebral palsy. Neural Plast 2005; 12: 197 203.

5. Donker SF, Ledebt A, Roerdink M, Savelsbergh GJ, Beek PJ. Children with cerebral palsy

DOI: http://dx.doi.org/10.4314/ejhs.v27i1.3 
exhibit greater or more regular postural sway than typically developing children. Exp Brain Res 2008; 184:363-70.

6. Burtner PA, Woollacott MH, Craft GL and Roncesvalles MN. The capacity to adapt to changing balance threats: a comparison of children with cerebral palsy and typically developing children. Dev Neurorehabil 2007; 10: 249-260.

7. Bartlett DJ and Palisano RJ. A multivariate model of determinants of motor change for children with cerebral palsy. Phys Ther 2000; 80: $598-614$

8. Hamzat TK and Mordi EL. Impact of caring for children with cerebral palsy on the general health of their caregivers in an African community.Int J Rehabil Res 2007; 30: 191-194

9. Law M, Darrah J, Pollock N, Rosenbaum P, Russell D, Walter $\mathrm{S}$ et al. Focus on function- a randomized controlled trial comparing two rehabilitation interventions for young children with cerebral palsy. BMC Pediatr 2007 27: 3143

10. Taylor NF, Dodd KJ, Damiano DL. Progressive Resistance Exercise in Physical Therapy: A Summary of Systematic Reviews. Phys Ther 2005; 85: 1208-1223

11. Kloos AD and Heiss DG. Exercise for impaired balance. In: Kisner $\mathrm{C}$ and ColbyL. Therapeutic Exercise: Foundations and Techniques.5ed: Philadelphia, F.A Davis company 2007; p.101, 251- 261.

12. Lee AJ and Lin WH. Twelve-week biomechanical ankle platform system training on postural stability and ankle proprioception in subjects with unilateral functional ankle instability. Clin Biomech 2008; 23: 1065-1072.

13. Ratliffe, K.T. Clinical Paediatric Physical Therapy. St Louis, Mosby Inc.; 1998. 184-192.

14. Palisano R. Validation of a Model of Gross Motor Function for Children with Cerebral Palsy. Phys Ther (serial online). October 2000; 80: 974 - 985. Available from: Academic Search Complete, Ipswich, MA.

15. Berg K, Wood-Dauphinee S, Williams $\mathrm{J}$ and Gayton D. Measuring balance in the elderly: preliminary development of an instrument. Physiotherapy Canada 1989; 41: 304-311.

16. Bohannon R and Smith M. Interrater Reliability of a Modified Ashworth Scale. Phys Ther 1987; 67:206-207.
17. Williams EN, Carroll SG, Reddihough DS, Phillips BA, Galea MP. Investigation of the timed up and go test in children. Dev Med Child Neurol 2005; 47: 518-524.

18. Kisner C, Colby L. Therapeutic Exercise: Foundation and Techniques. 5th ed. Philadelphia, F. A. Davis Company 2007; 251261

19. Bundonis J. Pediatric strength training. Rehab Manag 2007; 20(3):22, 24.

20. Palisano RJ, Copeland WP and Galuppi BE. Performance of physical activities by adolescents with cerebral palsy. Phys Ther 2007; 87: 77-87.

21. Palisano RJ, Kang LJ, Chiarello LA, Orlin, M, Oeffinger D and Maggs J. Social and community participation of children and youth with cerebral palsy is associated with age and gross motor function classification. Phys Ther 2009; 89: 1304 - 1314.

22. Lee JH, Sung IY, Yoo JY. Therapeutic effects of strengthening exercise on gait function of cerebral palsy. Disable Rehabil 2008; 30(19): $1439-44$

23. Scianni A, Butler JM, Ada L, Teixeira-Salmela LF. Muscle strengthening is not effective in children and adolescents with cerebral palsy: a systematic review. Aust $J$ Physioth 2009;55(2):81-7.

24. Park EY. Kim WH. Meta-analysis of the effect of strengthening interventions in individuals with cerebral palsy. Res Dev Disabil 2014; 35(2): 239-49

25. Jung JW, Her JG, Ko J. Effect of strength training of ankle plantarflexors on selective voluntary motor control, gait parameters, and gross motor function of children with cerebral palsy. J Phys Ther Sci 2013; 25(10):1259-63.

26. Woollacott MH, Shumway-Cook A, Hutchinson $\mathrm{S}$, Ciol M, Price R and Kartin D. Effects of balance training on muscle activity used in recovery of stability in Children with cerebral palsy: a pilot study. Dev Med Child Neurol 2005; 47:455-451.

DOI: http://dx.doi.org/10.4314/ejhs.v27i1.3 